\title{
Oscillatory Behavior of Second-Order Half-Linear Neutral Differential Equations with Damping
}

\author{
Ercan Tunç and Adil Kaymaz \\ Tokat Gaziosmanpasa University \\ Department of Mathematics \\ 60240, Tokat, Turkey
}

\begin{abstract}
This paper discusses the oscillatory behavior of solutions to a class of secondorder half-linear neutral differential equations with a damping term. Some new sufficient conditions for all solutions to be oscillatory are given. Examples illustrating our results are also included.
\end{abstract}

AMS Subject Classifications: 34C10, 34K11, 34K40.

Keywords: Oscillation, second order, neutral differential equations, damping term.

\section{Introduction}

This paper deals with the oscillatory behavior of all solutions of the second-order halflinear neutral differential equation with a damping term

$$
\left(r(t)\left(z^{\prime}(t)\right)^{\alpha}\right)^{\prime}+p(t)\left(z^{\prime}(t)\right)^{\alpha}+q(t) f(t, x(\sigma(t)))=0, \quad t \geq t_{0}>0,
$$

where $z(t)=x(t)+h(t) x(\tau(t))$, and $\alpha \geq 1$ is the ratio of two positive odd integers. Throughout this paper, we always assume that the following conditions are satisfied:

(i) $p, q, r:\left[t_{0}, \infty\right) \rightarrow \mathbb{R}$ are continuous functions with $p(t) \geq 0, r(t)>0, q(t)>0$, and

$$
\int_{t_{0}}^{\infty}\left[\frac{1}{r(t)} \exp \left(-\int_{t_{0}}^{t} \frac{p(s)}{r(s)} d s\right)\right]^{1 / \alpha} d t=\infty ;
$$

Received May 3, 2019; Accepted August 7, 2019

Communicated by Sandra Pinelas 
(ii) $h:\left[t_{0}, \infty\right) \rightarrow \mathbb{R}$ is a continuous function with $h(t) \geq 1$, and $h(t) \not \equiv 1$ for large $t$;

(iii) $\tau, \sigma:\left[t_{0}, \infty\right) \rightarrow \mathbb{R}$ are continuous functions such that $\tau$ is strictly increasing, $\tau(t)<t$, and $\lim _{t \rightarrow \infty} \tau(t)=\lim _{t \rightarrow \infty} \sigma(t)=\infty ;$

(iv) $f(t, u):\left[t_{0}, \infty\right) \times \mathbb{R} \rightarrow \mathbb{R}$ is a continuous function such that $u f(t, u)>0$ for all $u \neq 0$ and there exists a positive constant $k$ such that

$$
f(t, u) / u^{\alpha} \geq k \text { for } u \neq 0 .
$$

The cases where

$$
\tau(t) \geq \sigma(t)
$$

and

$$
\tau(t) \leq \sigma(t)
$$

are considered.

By a solution of equation (1.1), we mean a function $x \in C\left(\left[t_{x}, \infty\right), \mathbb{R}\right)$ for some $t_{x} \geq t_{0}$ that has the properties $z \in C^{1}\left(\left[t_{x}, \infty\right), \mathbb{R}\right), r\left(z^{\prime}\right)^{\alpha} \in C^{1}\left(\left[t_{x}, \infty\right), \mathbb{R}\right)$, and satisfies (1.1) on $\left[t_{x}, \infty\right)$. We only consider those solutions of (1.1) that exist on some half-line $\left[t_{x}, \infty\right)$ and satisfy the condition

$$
\sup \{|x(t)|: T \leq t<\infty\}>0 \text { for any } T \geq t_{x} ;
$$

moreover, we tacitly assume that (1.1) possesses such solutions. Such a solution $x(t)$ of (1.1) is said to be oscillatory if it has arbitrarily large zeros on $\left[t_{x}, \infty\right)$, i.e., for any $t_{1} \in$ $\left[t_{x}, \infty\right)$ there exists $t_{2} \geq t_{1}$ such that $x\left(t_{2}\right)=0$; otherwise it is called nonoscillatory, i.e., if it is eventually positive or eventually negative. Equation (1.1) itself is termed oscillatory if all its solutions are oscillatory.

The oscillatory behavior of solutions to various classes of second order functional differential equations has been the object of research of a number of authors and many interesting results have been obtained. For some typical results, we refer the reader to [2-4,7,8,10-12,15-20,23] and the references cited therein as examples of recent results on this topic. However, results on the oscillatory behavior of solutions of second-order neutral differential equations with damping term are relatively scarce in the literature; some results can be found, for example, in [5,6,21,22]. It should be noted that although papers $[5,6,21,22]$ deal with second-order neutral differential equations with damping term, the results obtained in these papers except [22] cannot be applied to the case where $h(t) \rightarrow \infty$ as $t \rightarrow \infty$. Motivated by the above observations, here we wish to develop sufficient conditions for equation (1.1) to be oscillatory in the case where $h(t)>1$ and/or $h(t) \rightarrow \infty$ as $t \rightarrow \infty$. The results of the present paper are obtained by using an integral averaging technique due to Philos [13] (see also $[9,14]$ for the refined integral averaging technique) and can easily be extended to more general second-order nonlinear neutral differential equations with damping term. It is therefore hoped that the present paper will contribute significantly to the study of oscillatory behavior of solutions of second-order neutral differential equations with damping term. 


\section{Main Results}

In the following theorems, we establish new oscillation criteria for (1.1) by using the integral averaging technique due to Philos [13]. In order to present our theorems, following Philos [13], we first introduce the function class $\mathcal{P}$. Namely, let $D_{0}=$ $\left\{(t, s): t>s \geq t_{0}\right\}$ and $D=\left\{(t, s): t \geq s \geq t_{0}\right\}$. We say that the function $H \in$ $C(D, \mathbb{R})$ belongs to the class $\mathcal{P}$, denoted by $H \in \mathcal{P}$, if

(i) $H(t, t)=0$ for $t \geq t_{0}$, and $H(t, s)>0$ on $(t, s) \in D_{0}$;

(ii) $H$ has a continuous and nonpositive partial derivative on $D_{0}$ with respect to the second variable.

For notational purposes, we let

$$
A\left(t, t_{*}\right):=\int_{t_{*}}^{t} \frac{d s}{r^{1 / \alpha}(s)}, t_{*} \geq t_{0}
$$

for any positive function $\eta \in C^{1}\left(\left[t_{0}, \infty\right), \mathbb{R}\right)$,

$$
\xi(t)=\frac{\eta^{\prime}(t) r(t)-\eta(t) p(t)}{\eta(t) r(t)}
$$

and

$$
\psi\left(t, t_{*}\right):=\frac{1}{h\left(\tau^{-1}(t)\right)}\left(1-\frac{1}{h\left(\tau^{-1}\left(\tau^{-1}(t)\right)\right)} \frac{A\left(\tau^{-1}\left(\tau^{-1}(t)\right), t_{*}\right)}{A\left(\tau^{-1}(t), t_{*}\right)}\right), t_{*} \geq t_{0},
$$

where $\tau^{-1}$ is the inverse function of $\tau$. Throughout this section we assume that $\psi\left(t, t_{*}\right)>$ 0 for all sufficiently large $t$.

Our first main result is contained in the following theorem.

Theorem 2.1. Let conditions (i)-(iv), (1.2) and (1.3) hold, and let $h, H: D \rightarrow \mathbb{R}$ be continuous functions such that $H$ belongs to the class $\mathcal{P}$ and

$$
-\frac{\partial H}{\partial s}(t, s)=h(t, s) \sqrt{H(t, s)} \quad \text { for all }(t, s) \in D_{0} .
$$

If there exists a positive function $\eta \in C^{1}\left(\left[t_{0}, \infty\right), \mathbb{R}\right)$ such that, for some $\gamma \geq 1$,

$$
\limsup _{t \rightarrow \infty} \frac{1}{H(t, T)} \int_{T}^{t}\left[H(t, s) \Psi(s)-\frac{\gamma}{4 \alpha} \frac{\eta(s) r^{1 / \alpha}(s) \Phi(t, s)}{A^{\alpha-1}\left(s, t_{2}\right)}\right] d s=\infty,
$$

for all sufficiently large $t_{2} \in\left[t_{1}, \infty\right) \subseteq\left[t_{0}, \infty\right)$, and all $T>t_{2}$ with $\sigma(t)>t_{2}$ for all $t \geq T$, where

$$
\Psi(t)=k \eta(t) q(t) \psi^{\alpha}\left(\sigma(t), t_{2}\right) \frac{A^{\alpha}\left(\tau^{-1}(\sigma(t)), t_{2}\right)}{A^{\alpha}\left(t, t_{2}\right)}
$$

and

$$
\Phi(t, s)=(-h(t, s)+\xi(s) \sqrt{H(t, s)})^{2},
$$

then every solution of (1.1) is oscillatory. 
Proof. Let $x(t)$ be a nonoscillatory solution of (1.1). Without loss of generality, we may assume that there exists $t_{1} \in\left[t_{0}, \infty\right)$ such that $x(t)>0, x(\tau(t))>0$, and $x(\sigma(t))>0$ for $t \geq t_{1}$. If $x(t)$ is eventually negative, the proof is similar, so we omit the details of that case here, as well as in the remaining proofs in this paper. Then, it follows from (1.1) that

$$
\left(r(t)\left(z^{\prime}(t)\right)^{\alpha}\right)^{\prime}+p(t)\left(z^{\prime}(t)\right)^{\alpha}+k q(t) x^{\alpha}(\sigma(t)) \leq 0
$$

and so

$$
\left(r(t)\left(z^{\prime}(t)\right)^{\alpha}\right)^{\prime}+p(t)\left(z^{\prime}(t)\right)^{\alpha}<0 \text { for } t \geq t_{1} .
$$

Letting $v(t)=r(t)\left(z^{\prime}(t)\right)^{\alpha}$, it follows from (2.6) that

$$
v^{\prime}(t)+\frac{p(t)}{r(t)} v(t)<0 \text { for } t \geq t_{1},
$$

which implies

$$
\left(\exp \left(\int_{t_{1}}^{t} \frac{p(s)}{r(s)} d s\right) v(t)\right)^{\prime}<0 \text { for } t \geq t_{1}
$$

and so, $v(t) \exp \left(\int_{t_{1}}^{t} \frac{p(s)}{r(s)} d s\right)$ is decreasing and eventually does not change its sign, say on $\left[t_{2}, \infty\right)$ for some $t_{2} \geq t_{1}$. Therefore, $z^{\prime}(t)$ eventually has a fixed sign on $\left[t_{2}, \infty\right)$, and so we have two cases to consider: (I) $z^{\prime}(t)>0$ for $t \geq t_{2}$ or (II) $z^{\prime}(t)<0$ for $t \geq t_{2}$.

We first assume that case (I) holds. It then follows from (2.5) and the definition of $z$ that

$$
z(t)>0, z^{\prime}(t)>0, \text { and }\left(r(t)\left(z^{\prime}(t)\right)^{\alpha}\right)^{\prime}<0 \text { for } t \geq t_{2},
$$

from which, we see that

$$
z(t)=z\left(t_{2}\right)+\int_{t_{2}}^{t} \frac{1}{r^{1 / \alpha}(s)}\left(r(s)\left(z^{\prime}(s)\right)^{\alpha}\right)^{1 / \alpha} d s \geq r^{1 / \alpha}(t) z^{\prime}(t) A\left(t, t_{2}\right) .
$$

In view of (2.7), we have for all $t \geq t_{3}$ for $t_{3} \in\left(t_{2}, \infty\right)$ that

$$
\left(\frac{z(t)}{A\left(t, t_{2}\right)}\right)^{\prime}=\frac{r^{-1 / \alpha}(t)\left[r^{1 / \alpha}(t) z^{\prime}(t) A\left(t, t_{2}\right)-z(t)\right]}{A^{2}\left(t, t_{2}\right)} \leq 0,
$$

i.e., $z(t) / A\left(t, t_{2}\right)$ is nonincreasing for $t \geq t_{3}$.

From the definition of $z$ (see also inequality (8.6) in [1]), it follows that

$$
\begin{aligned}
x(t) & =\frac{1}{h\left(\tau^{-1}(t)\right)}\left[z\left(\tau^{-1}(t)\right)-x\left(\tau^{-1}(t)\right)\right] \\
& =\frac{z\left(\tau^{-1}(t)\right)}{h\left(\tau^{-1}(t)\right)}-\frac{\left[z\left(\tau^{-1}\left(\tau^{-1}(t)\right)\right)-x\left(\tau^{-1}\left(\tau^{-1}(t)\right)\right)\right]}{h\left(\tau^{-1}(t)\right) h\left(\tau^{-1}\left(\tau^{-1}(t)\right)\right)}
\end{aligned}
$$




$$
\geq \frac{z\left(\tau^{-1}(t)\right)}{h\left(\tau^{-1}(t)\right)}-\frac{1}{h\left(\tau^{-1}(t)\right) h\left(\tau^{-1}\left(\tau^{-1}(t)\right)\right)} z\left(\tau^{-1}\left(\tau^{-1}(t)\right)\right)
$$

Now $\tau(t)<t$ and $\tau$ is strictly increasing, so $\tau^{-1}$ is increasing and $\tau^{-1}(t)>t$. Thus,

$$
\tau^{-1}\left(\tau^{-1}(t)\right)>\tau^{-1}(t)
$$

and since $z(t) / A\left(t, t_{2}\right)$ is nonincreasing for $t \geq t_{3}$, we have

$$
\frac{A\left(\tau^{-1}\left(\tau^{-1}(t)\right), t_{2}\right) z\left(\tau^{-1}(t)\right)}{A\left(\tau^{-1}(t), t_{2}\right)} \geq z\left(\tau^{-1}\left(\tau^{-1}(t)\right)\right) .
$$

Substituting the last inequality into (2.8) yields

$$
x(t) \geq \psi\left(t, t_{2}\right) z\left(\tau^{-1}(t)\right) \text { for } t \geq t_{3} .
$$

Since $\lim _{t \rightarrow \infty} \sigma(t)=\infty$, we can choose $t_{4} \geq t_{3}$ such that $\sigma(t) \geq t_{3}$ for all $t \geq t_{4}$. Thus, it follows from (2.9) that

$$
x(\sigma(t)) \geq \psi\left(\sigma(t), t_{2}\right) z\left(\tau^{-1}(\sigma(t))\right) \text { for } t \geq t_{4} .
$$

Using (2.10) in (2.5) gives

$$
\left(r(t)\left(z^{\prime}(t)\right)^{\alpha}\right)^{\prime}+p(t)\left(z^{\prime}(t)\right)^{\alpha}+k q(t) \psi^{\alpha}\left(\sigma(t), t_{2}\right) z^{\alpha}\left(\tau^{-1}(\sigma(t))\right) \leq 0
$$

for $t \geq t_{4}$. Define the function $w$ by the Riccati type substitution

$$
w(t)=\eta(t) \frac{r(t)\left(z^{\prime}(t)\right)^{\alpha}}{z^{\alpha}(t)} \quad \text { for } t \geq t_{4} .
$$

Clearly, $w(t)>0$, and from (2.11)-(2.12), we see that

$$
w^{\prime}(t) \leq \xi(t) w(t)-k \eta(t) q(t) \psi^{\alpha}\left(\sigma(t), t_{2}\right) \frac{z^{\alpha}\left(\tau^{-1}(\sigma(t))\right)}{z^{\alpha}(t)}-\alpha \frac{w^{(1+\alpha) / \alpha}(t)}{(\eta(t) r(t))^{1 / \alpha}}
$$

for $t \geq t_{4}$. From (1.3) and the fact that $\tau$ is strictly increasing, we have

$$
\tau^{-1}(\sigma(t)) \leq t
$$

and since $z(t) / A\left(t, t_{2}\right)$ is nonincreasing on $\left[t_{4}, \infty\right) \subseteq\left[t_{3}, \infty\right)$, we get

$$
\frac{z\left(\tau^{-1}(\sigma(t))\right)}{z(t)} \geq \frac{A\left(\tau^{-1}(\sigma(t)), t_{2}\right)}{A\left(t, t_{2}\right)} .
$$

Using (2.14) in (2.13), we obtain

$$
w^{\prime}(t) \leq \xi(t) w(t)-k \eta(t) q(t) \psi^{\alpha}\left(\sigma(t), t_{2}\right) \frac{A^{\alpha}\left(\tau^{-1}(\sigma(t)), t_{2}\right)}{A^{\alpha}\left(t, t_{2}\right)}-\alpha \frac{w^{(1+\alpha) / \alpha}(t)}{(\eta(t) r(t))^{1 / \alpha}},
$$


which can be written as, for $t \geq t_{4}$,

$$
w^{\prime}(t) \leq \xi(t) w(t)-\Psi(t)-\frac{\alpha w^{1 / \alpha-1}(t)}{(\eta(t) r(t))^{1 / \alpha}} w^{2}(t) .
$$

In view of (2.7) and (2.12), for $t \geq t_{4}$ we have

$$
\begin{array}{r}
w^{\frac{1}{\alpha}-1}(t)=\left(\eta(t) r(t)^{\frac{1}{\alpha}-1}\left(\left(\frac{z^{\prime}(t)}{z(t)}\right)^{\alpha}\right)^{\frac{1}{\alpha}-1}=\left(\eta(t) r(t)^{\frac{1}{\alpha}-1}\left(\frac{z(t)}{z^{\prime}(t)}\right)^{\alpha-1}\right.\right. \\
\geq \eta^{\frac{1}{\alpha}-1}(t) A^{\alpha-1}\left(t, t_{2}\right) .
\end{array}
$$

Using (2.16) in (2.15), we arrive at

$$
w^{\prime}(t) \leq \xi(t) w(t)-\Psi(t)-\frac{\alpha A^{\alpha-1}\left(t, t_{2}\right)}{\eta(t) r^{1 / \alpha}(t)} w^{2}(t) .
$$

Multiplying (2.17) by $H(t, s)$ and integrating from $T$ to $t$, we have, for some $\gamma \geq 1$ and for all $t \geq T \geq t_{4}$,

$$
\begin{aligned}
\int_{T}^{t} H(t, s) \Psi(s) d s \leq & -\int_{T}^{t} H(t, s) w^{\prime}(s) d s+\int_{T}^{t} H(t, s) \xi(s) w(s) d s \\
& -\frac{\alpha}{\gamma} \int_{T}^{t} H(t, s) \frac{A^{\alpha-1}\left(s, t_{2}\right)}{\eta(s) r^{1 / \alpha}(s)} w^{2}(s) d s \\
& -\frac{\alpha(\gamma-1)}{\gamma} \int_{T}^{t} H(t, s) \frac{A^{\alpha-1}\left(s, t_{2}\right)}{\eta(s) r^{1 / \alpha}(s)} w^{2}(s) d s
\end{aligned}
$$

An integrating by parts yields

$$
\begin{aligned}
\int_{T}^{t} H(t, s) w^{\prime}(s) d s & =\left.H(t, s) w(s)\right|_{T} ^{t}-\int_{T}^{t} \frac{\partial H}{\partial s}(t, s) w(s) d s \\
& =-H(t, T) w(T)-\int_{T}^{t} \frac{\partial H}{\partial s}(t, s) w(s) d s .
\end{aligned}
$$

Substituting (2.19) into (2.18) and taking (2.1) into account yields

$$
\begin{aligned}
\int_{T}^{t} H(t, s) \Psi(s) d s & \leq H(t, T) w(T) \\
& +\int_{T}^{t}[-h(t, s) \sqrt{H(t, s)}+H(t, s) \xi(s)] w(s) d s \\
& -\frac{\alpha}{\gamma} \int_{T}^{t} H(t, s) \frac{A^{\alpha-1}\left(s, t_{2}\right)}{\eta(s) r^{1 / \alpha}(s)} w^{2}(s) d s \\
& -\frac{\alpha(\gamma-1)}{\gamma} \int_{T}^{t} H(t, s) \frac{A^{\alpha-1}\left(s, t_{2}\right)}{\eta(s) r^{1 / \alpha}(s)} w^{2}(s) d s .
\end{aligned}
$$


Completing the square with respect to $w$, it follows from (2.20) that

$$
\begin{aligned}
\int_{T}^{t}[H(t, s) \Psi(s) & \left.-\frac{\gamma}{4 \alpha} \frac{\eta(s) r^{1 / \alpha}(s) \Phi(t, s)}{A^{\alpha-1}\left(s, t_{2}\right)}\right] d s \leq H(t, T) w(T) \\
& -\frac{\alpha(\gamma-1)}{\gamma} \int_{T}^{t} H(t, s) \frac{A^{\alpha-1}\left(s, t_{2}\right)}{\eta(s) r^{1 / \alpha}(s)} w^{2}(s) d s .
\end{aligned}
$$

So, for every $t \geq t_{4}$, we obtain

$$
\int_{t_{4}}^{t}\left[H(t, s) \Psi(s)-\frac{\gamma}{4 \alpha} \frac{\eta(s) r^{1 / \alpha}(s) \Phi(t, s)}{A^{\alpha-1}\left(s, t_{2}\right)}\right] d s \leq H\left(t, t_{4}\right) w\left(t_{4}\right),
$$

which contradicts (2.2).

Next, we consider case (II). Letting $u(t)=r(t)\left(-z^{\prime}(t)\right)^{\alpha}>0$ for $t \geq t_{2}$, it follows from (1.1) that

$$
u^{\prime}(t)+\frac{p(t)}{r(t)} u(t) \geq 0 \quad \text { for } t \geq t_{2} .
$$

Integrating this relation from $t_{2}$ to $t$, we obtain

$$
u(t) \geq u\left(t_{2}\right) \exp \left(-\int_{t_{2}}^{t} \frac{p(s)}{r(s)} d s\right)
$$

from which we have

$$
z^{\prime}(t) \leq r^{1 / \alpha}\left(t_{2}\right) z^{\prime}\left(t_{2}\right)\left[\frac{1}{r(t)} \exp \left(-\int_{t_{2}}^{t} \frac{p(s)}{r(s)} d s\right)\right]^{1 / \alpha} .
$$

Integrating (2.22) from $t_{2}$ to $t$ and taking (1.2) into account, we see that

$$
z(t) \leq z\left(t_{2}\right)+r^{1 / \alpha}\left(t_{2}\right) z^{\prime}\left(t_{2}\right) \int_{t_{2}}^{t}\left[\frac{1}{r(s)} \exp \left(-\int_{t_{2}}^{s} \frac{p(u)}{r(u)} d u\right)\right]^{1 / \alpha} d s \rightarrow-\infty
$$

as $t \rightarrow \infty$, which contradicts the positivity of $z(t)$ and completes the proof.

The following oscillation criterion follows immediately from Theorem 2.1.

Corollary 2.2. Let the assumptions of Theorem 2.1 be satisfied except that condition (2.2) is replaced by

$$
\limsup _{t \rightarrow \infty} \frac{1}{H(t, T)} \int_{T}^{t} k^{-1} H(t, s) \Psi(s) d s=\infty
$$

and

$$
\limsup _{t \rightarrow \infty} \frac{1}{H(t, T)} \int_{T}^{t} \frac{\eta(s) r^{1 / \alpha}(s) \Phi(t, s)}{A^{\alpha-1}\left(s, t_{2}\right)} d s<\infty .
$$

Then equation (1.1) is oscillatory. 
Theorem 2.3. Suppose that conditions (i)-(iv), (1.2) and (1.3) are satisfied. Let $H$ and $h$ be as in Theorem 2.1 such that (2.1) holds, and

$$
0<\inf _{s \geq t_{0}}\left\{\liminf _{t \rightarrow \infty} \frac{H(t, s)}{H\left(t, t_{0}\right)}\right\} \leq \infty .
$$

If there exist functions $\phi \in C\left(\left[t_{0}, \infty\right), \mathbb{R}\right)$ and $\eta \in C^{1}\left(\left[t_{0}, \infty\right),(0, \infty)\right)$ such that, for some $\gamma>1$,

$$
\limsup _{t \rightarrow \infty} \frac{1}{H(t, T)} \int_{T}^{t}\left[H(t, s) \Psi(s)-\frac{\gamma}{4 \alpha} \frac{\eta(s) r^{1 / \alpha}(s) \Phi(t, s)}{A^{\alpha-1}\left(s, t_{2}\right)}\right] d s \geq \phi(T)
$$

and

$$
\int_{T}^{\infty} \frac{A^{\alpha-1}\left(s, t_{2}\right)}{\eta(s) r^{1 / \alpha}(s)} \phi_{+}^{2}(s) d s=\infty
$$

for all sufficiently large $t_{2} \in\left[t_{1}, \infty\right) \subseteq\left[t_{0}, \infty\right)$, and all $T>t_{2}$ with $\sigma(t)>t_{2}$ for all $t \geq T$, where $\Psi(s)$ and $\Phi(t, s)$ are as in Theorem 2.1 , and $\phi_{+}(t)=\max \{\phi(t), 0\}$, then every solution of (1.1) is oscillatory.

Proof. Let $x(t)$ be a nonoscillatory solution of equation (1.1), say $x(t)>0, x(\tau(t))>$ 0 , and $x(\sigma(t))>0$ for $t \geq t_{1}$ for some $t_{1} \in\left[t_{0}, \infty\right)$. Proceeding as in the proof of Theorem 2.1, we again have the two cases to consider: (I) $z^{\prime}(t)>0$ for $t \geq t_{2}$ or (II) $z^{\prime}(t)<0$ for $t \geq t_{2}$. If case (II) holds, proceeding exactly as in the proof of Theorem 2.1, we obtain a contradiction to the positivity of $z$.

Next, assume that case (I) holds. Proceeding as in the proof of Theorem 2.1, we again arrive at (2.21), which can be written as, for $t>T \geq t_{4}$,

$$
\begin{array}{r}
\frac{1}{H(t, T)} \int_{T}^{t}\left[H(t, s) \Psi(s)-\frac{\gamma}{4 \alpha} \frac{\eta(s) r^{1 / \alpha}(s) \Phi(t, s)}{A^{\alpha-1}\left(s, t_{2}\right)}\right] d s \\
\leq w(T)-\frac{1}{H(t, T)} \int_{T}^{t} \frac{\alpha(\gamma-1)}{\gamma} \frac{H(t, s) A^{\alpha-1}\left(s, t_{2}\right)}{\eta(s) r^{1 / \alpha}(s)} w^{2}(s) d s .
\end{array}
$$

From (2.28), we see that

$$
\begin{aligned}
& \limsup _{t \rightarrow \infty} \frac{1}{H(t, T)} \int_{T}^{t}\left[H(t, s) \Psi(s)-\frac{\gamma}{4 \alpha} \frac{\eta(s) r^{1 / \alpha}(s) \Phi(t, s)}{A^{\alpha-1}\left(s, t_{2}\right)}\right] d s \\
\leq & w(T)-\liminf _{t \rightarrow \infty} \frac{1}{H(t, T)} \int_{T}^{t} \frac{\alpha(\gamma-1)}{\gamma} \frac{H(t, s) A^{\alpha-1}\left(s, t_{2}\right)}{\eta(s) r^{1 / \alpha}(s)} w^{2}(s) .
\end{aligned}
$$

In view of (2.26), it follows from (2.29) that

$$
w(T) \geq \phi(T)+\liminf _{t \rightarrow \infty} \frac{1}{H(t, T)} \int_{T}^{t} \frac{\alpha(\gamma-1)}{\gamma} \frac{H(t, s) A^{\alpha-1}\left(s, t_{2}\right)}{\eta(s) r^{1 / \alpha}(s)} w^{2}(s) d s
$$


for all $t>T \geq t_{4}$ and for any $\gamma>1$. Thus, for all $T \geq t_{4}$,

$$
w(T) \geq \phi(T)
$$

and

$$
\liminf _{t \rightarrow \infty} \frac{1}{H\left(t, t_{4}\right)} \int_{t_{4}}^{t} \frac{H(t, s) A^{\alpha-1}\left(s, t_{2}\right)}{\eta(s) r^{1 / \alpha}(s)} w^{2}(s) d s \leq \frac{\gamma\left(w\left(t_{4}\right)-\phi\left(t_{4}\right)\right)}{\alpha(\gamma-1)}<\infty .
$$

Now, we claim that

$$
\int_{t_{4}}^{\infty} \frac{A^{\alpha-1}\left(s, t_{2}\right)}{\eta(s) r^{1 / \alpha}(s)} w^{2}(s) d s<\infty .
$$

Suppose the contrary, that is,

$$
\int_{t_{4}}^{\infty} \frac{A^{\alpha-1}\left(s, t_{2}\right)}{\eta(s) r^{1 / \alpha}(s)} w^{2}(s) d s=\infty .
$$

By (2.25), there exists a constant $\varepsilon>0$ such that

$$
\inf _{s \geq t_{0}}\left\{\liminf _{t \rightarrow \infty} \frac{H(t, s)}{H\left(t, t_{0}\right)}\right\}>\varepsilon .
$$

On the other hand, by virtue of (2.34), for any positive number $\delta$, there exists a $t_{5}>t_{4}$ such that

$$
\int_{t_{4}}^{t} \frac{A^{\alpha-1}\left(s, t_{2}\right)}{\eta(s) r^{1 / \alpha}(s)} w^{2}(s) d s \geq \frac{\delta}{\varepsilon} \quad \text { for all } t \geq t_{5} .
$$

Using integration by parts and taking (2.36) into account, we conclude that, for all $t \geq t_{5}$,

$$
\begin{aligned}
& \frac{1}{H\left(t, t_{4}\right)} \int_{t_{4}}^{t} H(t, s) \frac{A^{\alpha-1}\left(s, t_{2}\right)}{\eta(s) r^{1 / \alpha}(s)} w^{2}(s) d s \\
& =\frac{1}{H\left(t, t_{4}\right)} \int_{t_{4}}^{t} H(t, s) d\left[\int_{t_{4}}^{s} \frac{A^{\alpha-1}\left(\xi, t_{2}\right)}{\eta(\xi) r^{1 / \alpha}(\xi)} w^{2}(\xi) d \xi\right] \\
& =\frac{1}{H\left(t, t_{4}\right)} \int_{t_{4}}^{t}\left[\int_{t_{4}}^{s} \frac{A^{\alpha-1}\left(\xi, t_{2}\right)}{\eta(\xi) r^{1 / \alpha}(\xi)} w^{2}(\xi) d \xi\right]\left[-\frac{\partial H(t, s)}{\partial s}\right] d s \\
& \geq \frac{\delta}{\varepsilon} \frac{1}{H\left(t, t_{4}\right)} \int_{t_{5}}^{t}\left[-\frac{\partial H(t, s)}{\partial s}\right] d s \\
& =\frac{\delta}{\varepsilon} \frac{H\left(t, t_{5}\right)}{H\left(t, t_{4}\right)} \geq \frac{\delta}{\varepsilon} \frac{H\left(t, t_{5}\right)}{H\left(t, t_{0}\right)} .
\end{aligned}
$$

It follows from (2.35) that

$$
\liminf _{t \rightarrow \infty} \frac{H(t, s)}{H\left(t, t_{0}\right)}>\varepsilon>0,
$$


and hence there exists a $t_{6} \geq t_{5}$ such that

$$
\frac{H\left(t, t_{5}\right)}{H\left(t, t_{0}\right)} \geq \varepsilon \quad \text { for all } t \geq t_{6}
$$

From the latter inequality and (2.37), we see that

$$
\frac{1}{H\left(t, t_{4}\right)} \int_{t_{4}}^{t} H(t, s) \frac{A^{\alpha-1}\left(s, t_{2}\right)}{\eta(s) r^{1 / \alpha}(s)} w^{2}(s) d s \geq \delta \quad \text { for } t \geq t_{6} .
$$

Since $\delta$ is an arbitrary positive constant, we have

$$
\liminf _{t \rightarrow \infty} \frac{1}{H\left(t, t_{4}\right)} \int_{t_{4}}^{t} H(t, s) \frac{A^{\alpha-1}\left(s, t_{2}\right)}{\eta(s) r^{1 / \alpha}(s)} w^{2}(s) d s=\infty,
$$

which contradicts (2.32). Thus, (2.33) should hold, and so, by (2.31) we have

$$
\int_{t_{4}}^{\infty} \frac{A^{\alpha-1}\left(s, t_{2}\right)}{\eta(s) r^{1 / \alpha}(s)} \phi_{+}^{2}(s) d s \leq \int_{t_{4}}^{\infty} \frac{A^{\alpha-1}\left(s, t_{2}\right)}{\eta(s) r^{1 / \alpha}(s)} w^{2}(s) d s<\infty,
$$

which contradicts (2.27). This proves the theorem.

Theorem 2.4. Let all conditions of Theorem 2.3 be satisfied except that condition (2.26) be replaced with

$$
\liminf _{t \rightarrow \infty} \frac{1}{H(t, T)} \int_{T}^{t}\left[H(t, s) \Psi(s)-\frac{\gamma}{4 \alpha} \frac{\eta(s) r^{1 / \alpha}(s) \Phi(t, s)}{A^{\alpha-1}\left(s, t_{2}\right)}\right] d s \geq \phi(T) .
$$

Then, every solution of (1.1) is oscillatory.

Proof. The proof follows from the fact that

$$
\begin{aligned}
\phi(T) & \leq \liminf _{t \rightarrow \infty} \frac{1}{H(t, T)} \int_{T}^{t}\left[H(t, s) \Psi(s)-\frac{\gamma}{4 \alpha} \frac{\eta(s) r^{1 / \alpha}(s) \Phi(t, s)}{A^{\alpha-1}\left(s, t_{2}\right)}\right] d s \\
& \leq \limsup _{t \rightarrow \infty} \frac{1}{H(t, T)} \int_{T}^{t}\left[H(t, s) \Psi(s)-\frac{\gamma}{4 \alpha} \frac{\eta(s) r^{1 / \alpha}(s) \Phi(t, s)}{A^{\alpha-1}\left(s, t_{2}\right)}\right] d s,
\end{aligned}
$$

and so we omit the details.

Next, we give oscillation results in the case when (1.4) holds.

Theorem 2.5. Let conditions (i)-(iv), (1.2) and (1.4) be fulfilled, and let $H$ and $h$ be as in Theorem 2.1 such that (2.1) holds. If there exists a positive function $\eta \in$ $C^{1}\left(\left[t_{0}, \infty\right), \mathbb{R}\right)$ such that, for some $\gamma \geq 1$,

$$
\limsup _{t \rightarrow \infty} \frac{1}{H(t, T)} \int_{T}^{t}\left[H(t, s) \Omega(s)-\frac{\gamma}{4 \alpha} \frac{\eta(s) r^{1 / \alpha}(s) \Phi(t, s)}{A^{\alpha-1}\left(s, t_{2}\right)}\right] d s=\infty
$$


for all sufficiently large $t_{2} \in\left[t_{1}, \infty\right) \subseteq\left[t_{0}, \infty\right)$, and all $T>t_{2}$ with $\sigma(t)>t_{2}$ for all $t \geq T$, where

$$
\Omega(t)=k \eta(t) q(t) \psi^{\alpha}\left(\sigma(t), t_{2}\right),
$$

and $\Phi(t, s)$ is as in (2.4), then every solution of (1.1) is oscillatory.

Proof. Let $x(t)$ be a nonoscillatory solution of (1.1) with $x(t)>0, x(\tau(t))>0$, and $x(\sigma(t))>0$ for $t \geq t_{1}$ for some $t_{1} \in\left[t_{0}, \infty\right)$. Proceeding as in the proof of Theorem 2.1, we again have two cases to consider: (I) $z^{\prime}(t)>0$ for $t \geq t_{2}$ or (II) $z^{\prime}(t)<0$ for $t \geq t_{2}$. If case (II) holds, as in the proof of Theorem 2.1, we contradict the positivity of $z(t)$.

If case (I) holds, then, as in the proof of Theorem 2.1, we again arrive at (2.13) for $t \geq t_{4}$. From (1.4) and the fact that $\tau$ is strictly increasing, we have

$$
\tau^{-1}(\sigma(t)) \geq t
$$

and since $z$ is increasing, we obtain

$$
\frac{z\left(\tau^{-1}(\sigma(t))\right)}{z(t)} \geq 1
$$

Using (2.45) in (2.13) yields

$$
w^{\prime}(t) \leq \xi(t) w(t)-k \eta(t) q(t) \psi^{\alpha}\left(\sigma(t), t_{2}\right)-\alpha \frac{w^{(1+\alpha) / \alpha}(t)}{(\eta(t) r(t))^{1 / \alpha}} .
$$

The remainder of the proof is similar to the first part of the proof of Theorem 2.1 and hence is omitted.

Corollary 2.6. The conclusion of Theorem 2.5 remains intact if assumption (2.43) is replaced by the two conditions

$$
\limsup _{t \rightarrow \infty} \frac{1}{H(t, T)} \int_{T}^{t} k^{-1} H(t, s) \Omega(s) d s=\infty,
$$

and (2.24).

Theorem 2.7. Suppose that conditions (i)-(iv), (1.2) and (1.4) are satisfied. Let H and $h$ be as in Theorem 2.1 such that (2.1) and (2.25) hold. If there exist functions $\phi \in C\left(\left[t_{0}, \infty\right), \mathbb{R}\right)$ and $\eta \in C^{1}\left(\left[t_{0}, \infty\right),(0, \infty)\right)$ such that (2.27) holds, and for some $\gamma>1$

$$
\limsup _{t \rightarrow \infty} \frac{1}{H(t, T)} \int_{T}^{t}\left[H(t, s) \Omega(s)-\frac{\gamma}{4 \alpha} \frac{\eta(s) r^{1 / \alpha}(s) \Phi(t, s)}{A^{\alpha-1}\left(s, t_{2}\right)}\right] d s \geq \phi(T),
$$

for all sufficiently large $t_{2} \in\left[t_{1}, \infty\right) \subseteq\left[t_{0}, \infty\right)$, and all $T>t_{2}$ with $\sigma(t)>t_{2}$ for all $t \geq T$, where $\Omega(t)$ is as in (2.44), then every solution of (1.1) is oscillatory. 
Proof. The proof follows from (2.45), (2.46) and Theorem 2.3, so we omit the details.

Theorem 2.8. Let all conditions of Theorem 2.7 be satisfied except that condition (2.48) be replaced with

$$
\liminf _{t \rightarrow \infty} \frac{1}{H(t, T)} \int_{T}^{t}\left[H(t, s) \Omega(s)-\frac{\gamma}{4 \alpha} \frac{\eta(s) r^{1 / \alpha}(s) \Phi(t, s)}{A^{\alpha-1}\left(s, t_{2}\right)}\right] d s \geq \phi(T) .
$$

Then, every solution of (1.1) is oscillatory.

Proof. The proof follows from (2.45), (2.46) and Theorem 2.4, so we omit the details.

\section{Examples}

We conclude this paper with the following examples to illustrate the above results. The first example is concerned with the case where $h(t) \rightarrow \infty$ as $t \rightarrow \infty$, and the second example is concerned with the case where $h$ is a constant function.

Example 3.1. Consider the half-linear neutral differential equation with damping

$$
\left(\left(z^{\prime}(t)\right)^{5}\right)^{\prime}+\frac{1}{t}\left(z^{\prime}(t)\right)^{5}+(t+1)^{4} x^{5}(t-1)=0, t \geq 2
$$

with $z(t)=x(t)+t x(t-2)$. Here we have $\alpha=5, \tau(t)=t-2, \sigma(t)=t-1, r(t)=1$, $p(t)=1 / t, q(t)=(t+1)^{4}, h(t)=t$, and $f(t, x(\sigma(t)))=x^{5}(t-1)$. It is easy to see that conditions (i)-(iv), (1.2) and (1.4) hold. Choosing $t_{2}=t_{1}=t_{0}=2$, we have

$$
\begin{gathered}
A\left(t, t_{2}\right)=A(t, 2)=t-2, \\
A\left(\tau^{-1}(t), t_{2}\right)=A(t+2,2)=t, \\
A\left(\tau^{-1}\left(\tau^{-1}(t)\right), t_{2}\right)=A(t+4,2)=t+2, \\
\psi\left(t, t_{2}\right)=\frac{1}{t+2}\left(1-\frac{t+2}{t(t+4)}\right)>0 \text { for } t \geq t_{0}=2 .
\end{gathered}
$$

Letting $H(t, s)=(t-s)^{2}$, we see that $H \in \mathcal{P}$ and $h(t, s)=2$. With $\eta(t)=t$, we see that $\xi(t)=0$, and conditions (2.47) and (2.24) become, for all $T \in(3, \infty)$,

$$
\begin{array}{r}
\limsup _{t \rightarrow \infty} \frac{1}{H(t, T)} \int_{T}^{t} k^{-1} H(t, s) \Omega(s) d s \geq \limsup _{t \rightarrow \infty} \frac{(2 / 5)^{5}}{(t-T)^{2}} \int_{T}^{t}(t-s)^{2} \frac{s}{s+1} d s \\
\geq \limsup _{t \rightarrow \infty} \frac{(2 / 5)^{5} T}{T+1} \frac{1}{(t-T)^{2}} \int_{T}^{t}(t-s)^{2} d s
\end{array}
$$




$$
=\limsup _{t \rightarrow \infty} \frac{(2 / 5)^{5} T\left(t^{3}-3 t^{2} T+3 t T^{2}-T^{3}\right)}{3(T+1)(t-T)^{2}}=\infty
$$

and

$$
\begin{array}{r}
\limsup _{t \rightarrow \infty} \frac{1}{H(t, T)} \int_{T}^{t} \frac{\eta(s) r^{1 / \alpha}(s) \Phi(t, s)}{A^{\alpha-1}\left(s, t_{2}\right)} d s=\limsup _{t \rightarrow \infty} \frac{1}{(t-T)^{2}} \int_{T}^{t} \frac{4 s}{(s-2)^{4}} d s \\
\leq \limsup _{t \rightarrow \infty} \frac{1}{(t-T)^{2}} \frac{4}{(T-2)^{4}} \int_{T}^{t} s d s \\
=\limsup _{t \rightarrow \infty} \frac{2\left(t^{2}-T^{2}\right)}{(T-2)^{4}(t-T)^{2}}=\frac{2}{(T-2)^{4}}<\infty
\end{array}
$$

i.e., conditions (2.47) and (2.24) hold. Thus, all conditions of Corollary 2.6 are satisfied, so equation (3.1) is oscillatory.

Example 3.2. Consider the half-linear neutral differential equation with damping

$$
\left(\frac{1}{t^{3}}\left(z^{\prime}(t)\right)^{3}\right)^{\prime}+\frac{1}{t^{4}}\left(z^{\prime}(t)\right)^{3}+t^{2} x^{3}(t / 2)=0, t \geq 2
$$

with $z(t)=x(t)+10 x(t-1)$. Here we have $\alpha=3, \tau(t)=t-1, \sigma(t)=t / 2$, $r(t)=1 / t^{3}, p(t)=1 / t^{4}, q(t)=t^{2}, h(t)=10$, and $f(t, x(\sigma(t)))=x^{3}(t / 2)$. It is easy to see that conditions (i)-(iv), (1.2) and (1.3) hold, and with $t_{2}=t_{1}=t_{0}=2$, we have

$$
\begin{gathered}
A\left(t, t_{2}\right)=A(t, 2)=\left(t^{2}-4\right) / 2, \\
A\left(\tau^{-1}(t), t_{2}\right)=A(t+1,2)=\left((t+1)^{2}-4\right) / 2, \\
A\left(\tau^{-1}\left(\tau^{-1}(t)\right), t_{2}\right)=A(t+2,2)=\left((t+2)^{2}-4\right) / 2, \\
\psi\left(t, t_{2}\right)=\frac{1}{10}\left(1-\frac{t(t+4)}{10(t-1)(t+3)}\right)>0 \text { for } t \geq t_{0}=2 .
\end{gathered}
$$

Letting $H(t, s)=(t-s)^{2}$, we see that $H \in \mathcal{P}$ and $h(t, s)=2$. With $\eta(t)=t$, we have $\xi(t)=0$, and as in Example 3.1, it is easy to see that conditions (2.23) and (2.24) hold. Thus, all conditions of Corollary 2.2 are satisfied, so equation (3.2) is oscillatory.

\section{References}

[1] R. P. Agarwal, S. R. Grace, and D. O'Regan, The oscillation of certain higher-order functional dfferential equations, Math. Comput. Model., 37 (2003), 705-728.

[2] B. Baculíková, T. Li, and J. Džurina, Oscillation theorems for second order neutral differential equations, Electron. J. Qual. Theory Differ. Equ., 2011 (2011), No. 74, $1-13$. 
[3] M. Bohner, S. R. Grace, and I. Jadlovská, Oscillation criteria for second-order neutral delay differential equations, Electron. J. Qual. Theory. Differ. Equ., 2017 (2017), No. 60, 1-12.

[4] S. R. Grace, J. Džurina, I. Jadlovská, and T. Li, An improved approach for studying oscillation of second-order neutral delay differential equations, J. Inequal. Appl., 2018 (2018), Article ID 193, 1-13.

[5] S. R. Grace, J. R. Graef, and E. Tunç, Oscillatory behavior of second order damped neutral differential equations with distributed deviating arguments, Miskolc Math. Notes, 18 (2017), 759-769.

[6] S. R. Grace and I. Jadlovská, Oscillation criteria for second-order neutral damped differential equations with delay argument, Dynamical Systems-Analytical and Computational Techniques, INTECH, Chapter 2, 2017, 31-53.

[7] T. Li , R. P. Agarwal, and M. Bohner, Some oscillation results for second-order neutral dyanmic equations, Hacet. J. Math. Stat., 41 (2012), 715-721.

[8] T. Li and Y. V. Rogovchenko, Oscillatory behavior of second-order nonlinear neutral differential equations, Abstr. Appl. Anal., 2014 (2014), Article ID 143614, 1-8.

[9] T. Li, Y. V. Rogovchenko, and S. Tang, Oscillation of second-order nonlinear differential equations with damping, Math. Slovaca, 64 (2014), 1227-1236.

[10] T. Li, B. Baculíková, and J. Džurina, Oscillatory behavior of second-order nonlinear neutral differential equations with distributed deviating arguments, Bound. Value Probl., 2014 (2014), No. 68, 1-15.

[11] T. Li and Y. V. Rogovchenko, Oscillation of second-order neutral differential equations, Math. Nachr., 288 (2015), 1150-1162.

[12] T. Li and Y. V. Rogovchenko, Oscillation criteria for second-order superlinear Emden-Fowler neutral differential equations, Monatsh. Math., 184 (2017), 489500 .

[13] Ch. G. Philos, Oscillation theorems for linear differential equations of second order, Arch. Math., 53 (1989), 482-492.

[14] Y. V. Rogovchenko and F. Tuncay, Oscillation theorems for a class of second order nonlinear differential equations with damping, Taiwan J. Math., 13 (2009), 19091928.

[15] S. H. Saker, Oscillation of second order neutral delay differential equations of Emden-Fowler type, Acta. Math. Hungar., 100 (2003), 37-62. 
[16] S. H. Saker, P. Y. H. Pang, and R. P. Agarwal, Oscillation theorems for second order nonlinear functional differential equations with damping, Dynam. Syst. Appl., 12 (2003), 307-322.

[17] S. H. Saker, R. P. Agarwal, and D. O'Regan, Oscillation of second-order damped dynamic equations on time scales, J. Math. Anal. Appl., 330 (2007), 1317-1337.

[18] S. S. Santra, S. Pinelas, and J. G. Dix, Necessary and sufficient conditions for the oscillation of solutions to second-order nonlinear differential equations with several delays, Global Journal of Mathematics, 12 (2018), 805-810.

[19] E. Thandapani, V. Piramanantham, and S. Pinelas, Oscillation criteria for secondorder neutral delay dynamic equations with mixed nonlinearities, Adv. Differ. Equ., 2011 (2011), Article ID 513757, 1-14.

[20] E. Tunç and J. R. Graef, Oscillation results for second order neutral dynamic equations with distributed deviating arguments, Dynam. Syst. Appl., 23 (2014), 289303.

[21] E. Tunç and S. R. Grace, On oscillatory and asymptotic behavior of a second-order nonlinear damped neutral differential equation, Int. J. Differ. Equ., 2016 (2016), Article ID 3746368, 1-8.

[22] E. Tunç and A. Kaymaz, On oscillation of second-order linear neutral differential equations with damping term, Dynam. Syst. Appl., 28 (2019), 289-301.

[23] J. S. W. Wong, Necessary and sufficient conditions for oscillation of second order neutral differential equations, J. Math. Anal. Appl., 252 (2000), 342-352. 
